\title{
Open-Loop Power Sharing of Three-Port DC-DC Resonant Converters
}

Y. Tran, F. Freijedo, and D. Dujic

This material is posted here with permission of the IEEE. Such permission of the IEEE does not in any way imply IEEE endorsement of any of EPFL's products or services. Internal or personal use of this material is permitted. However, permission to reprint / republish this material for advertising or promotional purposes or for creating new collective works for resale or redistribution must be obtained from the IEEE by writing to pubs-permissions@ieee. org. By choosing to view this document, you agree to all provisions of the copyright laws protecting it. 


\title{
Open-Loop Power Sharing of Three-Port DC-DC Resonant Converters
}

\author{
Yan-Kim Tran, Francisco D. Freijedo, Drazen Dujic \\ Power Electronics Laboratory - PEL \\ École Polytechnique Fédérale de Lausanne - EPFL \\ Lausanne CH-1015, Switzerland \\ yan-kim.tran@epfl.ch, francisco.freijedo@epfl.ch,drazen.dujic@epfl.ch
}

\begin{abstract}
Multi-port DC-DC converters are an attractive solution for highly flexible and efficient interface of different elements of DC grids (e.g., sources, loads, energy storage). This paper provides an analytic solution for the natural power sharing characteristics of a galvanically isolated three-port resonant DCDC converter. A DC-transformer operation mode is considered, with two ports actively transferring power to a third port. The mathematical model reveals that the power sharing characteristics are tightly dominated by the resonant tank parameters, even though they are also dependent on the differential voltage at the input terminals and the system losses. The accuracy of the proposed model is verified by an experimental validation on a lab-scale prototype.
\end{abstract}

Index Terms-MVDC, Multiport, DC-DC, LLC, Resonant Converter, Solid State Transformer.

\section{INTRODUCTION}

Emerging applications are considering direct current (DC) power distribution networks, as an efficient way of increasing operational efficiency and design flexibility [1]. Some applications such as marine electrical on-board distribution networks [2], [3], photovoltaic parks [4], offshore wind-farms [5], data centers [6], or distribution in urban areas [7], are already looking into DC grid alternatives. In all the aforementioned cases, an interface between the different part of the system operating at different voltages is required. Additionally, the integration of an increasing number of low voltage DC sources, loads and especially storage elements [8], motivates the development of new types of converters able to provide controllable and bidirectional power flow on top of galvanic isolation (safety reasons), high efficiency and reliability. One key degree of freedom offered by the DC technology is the design of medium or high frequency operated transformers in order to realize compact power conversion structures [9].

Among the possible solutions to interface different DC grids, the power electronics traction transformer (PETT) is one topology already demonstrated at an industrial level [10], [11]. The PETT structure comprises different power conversion stages associated to different features: voltage control by the grid side converter, voltage adaptation and isolation by the LLC-MFT stage and power control by the load side converter. The control features are associated to the two outer stages (grid side and load side) while the LLC resonant converter plays the role of a DC-transformer [10], [11]. Even if this structure has been developed for AC traction supply, it could be adopted for DC conversion with minor modifications. Lately, the Multiport Energy Gateway (MEG) concept extends the PETT one by including energy storage into the system [12], which is achieved by using a multiport MFT to interface different input power sources at the LLC stage.

Relevant works considering resonant multiport MFTs in the LLC resonant converter are reported [12]-[17]. According to [13], the resonant frequency has to be the same for all the ports, which implies a carefully design of the resonant tank and its splitting among the multiple ports as well as the operation at a common and fixed switching frequency, which makes frequency, duty-cycle and phase-shift control no more possible. The operation of a three-winding MFT based resonant converter with a single source port and two load ports is presented in [14] and demonstrates good load and cross regulations. Operation with two synchronous source ports and one load port are shown by the authors of [15]. The MEG structure, presented in [12], shows two main ports that interface two different MVDC-LVDC grids, while the third one interfaces LV storage elements (batteries or ultracapacitors). Despite the state-of-the-art shows the feasibility of the multiport LLC topologies, a systematic and rigorous mathematical analysis describing the unforced power sharing principles of multiport LLC conversion is not available in the literature. This systematic evaluation is needed to address the limits of the topology and define the design rules that permit to consider the technology for industrial application.

To cover the lack of analysis that may help in the elaboration of design rules, this paper presents a rigorous mathematical modeling of the three-port resonant DC-DC converter; the natural power sharing is derived and characterized considering two source ports transferring power to a third one (passive rectifier) in a natural/unforced manner [17]. For this study, the two additional regulation stages in Fig. 1, described in [16], are substituted by ideal DC buses. The source DC voltages are considered in addition to the resonant tanks and MFT parameters. From the piece-wise modeling of the system, a mathematical model that describes the power sharing rules, which aims to be a key tool for further industrial level designs, is derived. 


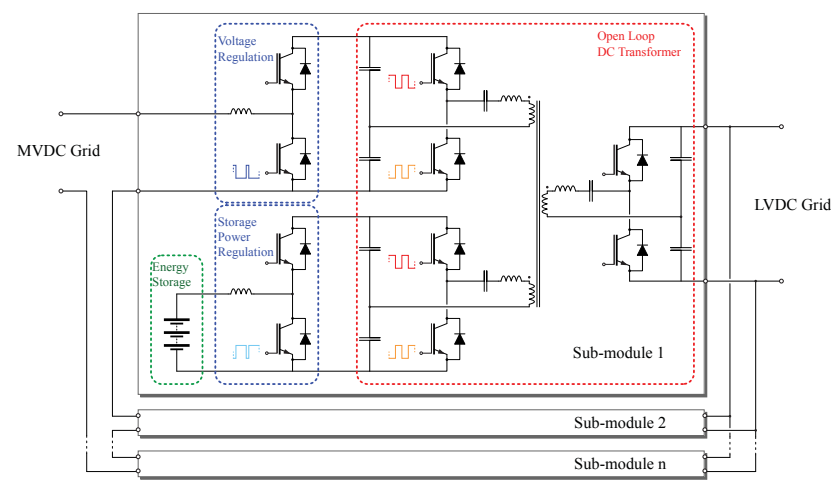

Fig. 1. MEG high power DC-DC-conversion as presented in [12]. The LLC resonant converter, in red, is operated as a DC-transformer (i.e. open loop modulation with $50 \%$ duty-cycle and constant frequency). The voltage and power of the different DC-buses have to be controlled by additional powe conversion stages, in blue. MEG is an evolution of the PETT concept [11] applied to multiport DC-DC conversion.

\section{Circuit And Problem Description}

Fig. 2 shows the topology under study, which includes a three-winding MFT with the number of turns $n_{1}, n_{2}, n_{3}$. The input ports ( 1 and 2 ) are equipped with two resonant tanks of identical resonant frequency $f_{\text {res }}$ composed by the capacitors $C_{1}$ and $C_{2}^{\prime}$ combined with the leakage inductance $L_{1}$ and $L_{2}^{\prime}$. Even though, in practice, part of the resonant tank would be located on the port 3 as well, this option is omitted for the sake of simplicity of the mathematical developments. The semiconductors of both input ports switch at the same fixed frequency with a constant duty-cycle of $50 \%$ and the same phase [17]. In order to benefit not only from ZVS [18] and reduced turn-off current on the primary switches but also from ZCS on the secondary diode rectifier, the switching frequency is set slightly below the resonant frequency $\left(f_{s w}<f_{\text {res }}\right)$ [19]. The DC bus capacitors $C_{D C}$ are sized much bigger than the resonant capacitors so, for the purpose of modelling, their voltage $V_{D C}$ can be considered constant; i.e., square-wave

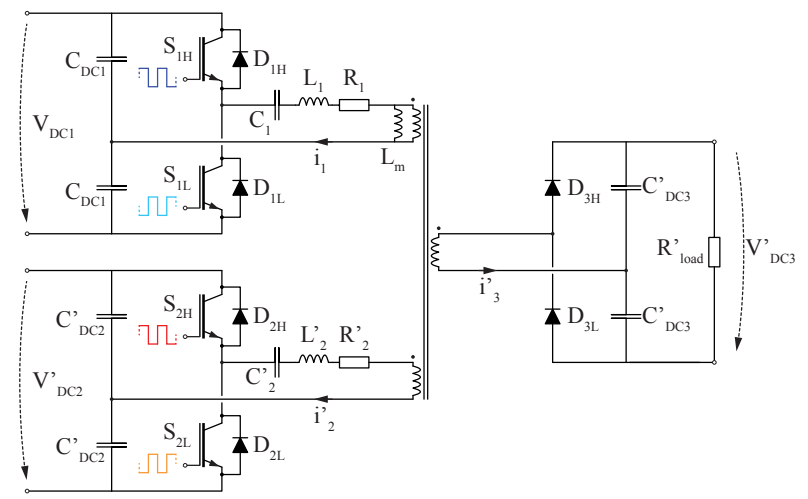

Fig. 2. Topology of a three-port resonant DC-DC converter operated with two active ports

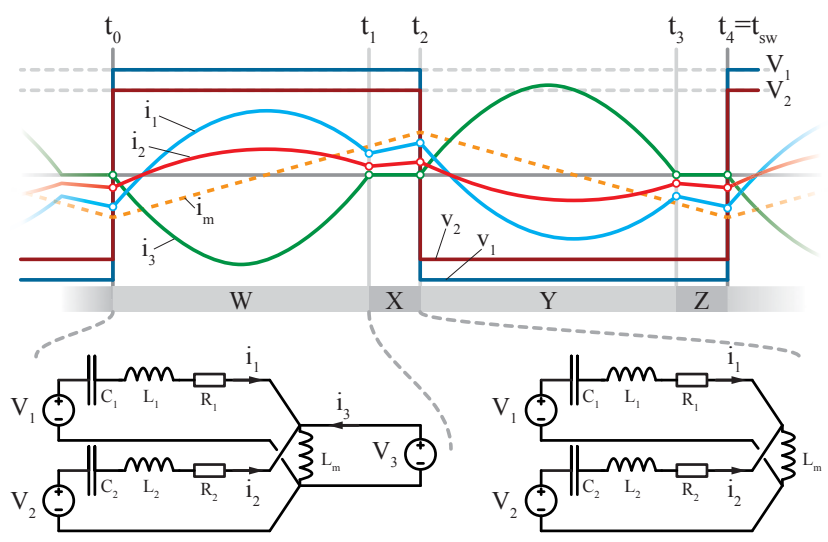

Fig. 3. Steady state voltage and current waveforms. During interval $W$, the equivalent circuit is simplified considering three DC voltage sources, two resonant tank and the magnetizing inductance. The voltage applied the magnetizing inductance terminals is clamped by $V_{3}$ which leads to the set of differential equations (1). During interval $X$, the diodes on the load port stop conducting and $V_{3}$ is disconnected. The equivalent circuit comprise only the two input voltage which leads to the set of differential equations (8).

voltage sources of amplitude $V_{1}=V_{D C_{1}} / 2$ and $V_{2}^{\prime}=V_{D C_{2}}^{\prime} / 2$ are considered.

The power transferred to the load port $\left(P_{\text {load }}=P_{3}\right)$ is the sum of both contributions $P_{1}$ and $P_{2}$ from each active ports. $L_{2}^{\prime}, C_{2}^{\prime}, V_{2}^{\prime}$ and $V_{3}^{\prime}$ are referred to the port 1 of the transformer and represented by $L_{2}=L_{2}^{\prime} n_{1}^{2} / n_{2}^{2}$, $C_{2}=C_{2}^{\prime} n_{2}^{2} / n_{1}^{2}, V_{2}=V_{2}^{\prime} n_{1} / n_{2}$ and $V_{3}=V_{3}^{\prime} n_{1} / n_{3}$. Then for the sake of simplicity of the theoretical approach, a lossless converter without parasitic resistance $\left(R_{1}=R_{2}=0\right)$ is considered firstly. The converter is sized to be operated in halfcycle discontinuous mode (HC-DCM) [20] over its complete operating range. Thus two main intervals can be identified per half-period and are depicted in Fig. 3. During interval $W$ the two active ports are delivering power to the load as well as the magnetizing current $i_{m}$; the voltage seen at the magnetizing inductance terminals is clamped by the voltage on the load port due to the conduction of the diodes [16], [17]. When the output diode rectifier stops conducing, interval $X$ starts. The two active ports are only supporting $i_{m}$, and the magnetizing inductance terminals are no longer clamped, while the load is supported by the output capacitors. Intervals $Y$ and $Z$ are the same as $W$ and $X$ respectively with opposite voltage and current polarity. The circuit time-domain equations should be calculated for each interval. For the interval $W$, these are:

$$
\begin{aligned}
v_{1}(t) & =v_{C_{1}}^{W}(t)+L_{1} \frac{d i_{1}^{W}(t)}{d t}+v_{3}(t) \\
i_{1}(t) & =C_{1} \frac{d v_{C_{1}}^{W}(t)}{d t} \\
v_{2}(t) & =v_{C_{2}}^{W}(t)+L_{2} \frac{d i_{2}^{W}(t)}{d t}+v_{3}(t) \\
i_{2}(t) & =C_{2} \frac{d v_{C_{2}}^{W}(t)}{d t} .
\end{aligned}
$$


and for the third port, they are given by:

$$
\begin{aligned}
v_{3}(t) & =L_{m} \frac{d i_{m}^{W}(t)}{d t} \\
i_{3}^{W}(t) & =-i_{1}^{W}(t)-i_{2}^{W}(t)+i_{m}^{W}(t) .
\end{aligned}
$$

The Laplace transform with initial conditions is considered to obtain time-domain expressions. From the step response at $t=0$, and assuming ideal voltage sources, of amplitudes $V_{1}, V_{2}$ and $V_{3}$, respectively:

$$
\begin{aligned}
& \frac{V_{1}}{s}=\frac{v_{C_{1}}\left(t_{0}\right)}{s}+\frac{1}{s C_{1}} i_{1}^{W}(s)+s L_{1} i_{1}^{W}(s)-L_{1} i_{1}\left(t_{0}\right)+\frac{V_{3}}{s} \\
& \frac{V_{2}}{s}=\frac{v_{C_{2}}\left(t_{0}\right)}{s}+\frac{1}{s C_{2}} i_{2}^{W}(s)+s L_{2} i_{2}^{W}(s)-L_{2} i_{2}\left(t_{0}\right)+\frac{V_{3}}{s} \\
& i_{m}^{W}(s)=\frac{V_{3}}{s^{2} L_{m}}+\frac{i_{1}\left(t_{0}\right)+i_{2}\left(t_{0}\right)}{s} .
\end{aligned}
$$

Expressions for $i_{1}(s)$ and $i_{2}(s)$ can be simplified by introducing $\omega^{2}=1 /\left(L_{1} C_{1}\right)=1 /\left(L_{2} C_{2}\right)$, which gives (for $k=1,2$ ):

$$
i_{k}^{W}(s)=\left(\frac{V_{k}-v_{C_{k}}\left(t_{0}\right)-V_{3}}{\omega L_{k}}\right) \frac{\omega}{\omega^{2}+s^{2}}+i_{k}\left(t_{0}\right) \frac{s}{\omega^{2}+s^{2}}
$$

Back in time domain, it yields:

$$
i_{k}^{W}(t)=i_{k}\left(t_{0}\right) \cos (\omega t)+\left(\frac{V_{k}-v_{C_{k}}\left(t_{0}\right)-V_{3}}{\omega L_{k}}\right) \sin (\omega t)
$$

and

$$
i_{m}^{W}(t)=i_{1}\left(t_{0}\right)+i_{2}\left(t_{0}\right)+\frac{V_{3}}{L_{m}} t .
$$

For the interval $X$, the voltage equations are:

$$
\begin{aligned}
& V_{1}=v_{C_{1}}^{X}(t)+L_{1} \frac{d i_{1}^{X}(t)}{d t}+L_{m} \frac{d\left(i_{1}^{X}(t)+i_{2}^{X}(t)\right)}{d t} \\
& V_{2}=v_{C_{2}}^{X}(t)+L_{2} \frac{d i_{2}^{X}(t)}{d t}+L_{m} \frac{d\left(i_{1}^{X}(t)+i_{2}^{X}(t)\right)}{d t} .
\end{aligned}
$$

Using Laplace transform and the initial conditions:

$\frac{V_{1}}{s}=\frac{v_{C_{1}}\left(t_{1}\right)}{s}+\frac{1}{s C_{1}} i_{1}^{X}(s)+s L_{1} i_{1}^{X}(s)-L_{1} i_{1}\left(t_{1}\right)+v_{m}^{X}(s)$ $\frac{V_{2}}{s}=\frac{v_{C_{2}}\left(t_{1}\right)}{s}+\frac{1}{s C_{2}} i_{2}^{X}(s)+s L_{2} i_{2}^{X}(s)-L_{2} i_{2}\left(t_{1}\right)+v_{m}^{X}(s)$.

And subsequently, it yields:

$$
\begin{aligned}
& i_{1}^{X}(s)=\left(\frac{V_{1}}{s}-\frac{v_{C_{1}}\left(t_{1}\right)}{s}+L_{1} i_{1}\left(t_{1}\right)-v_{m}^{X}(s)\right) \frac{1}{\frac{1}{s C_{1}}+s L_{1}} \\
& i_{2}^{X}(s)=\left(\frac{V_{2}}{s}-\frac{v_{C_{2}}\left(t_{1}\right)}{s}+L_{2} i_{2}\left(t_{1}\right)-v_{m}^{X}(s)\right) \frac{1}{\frac{1}{s C_{2}}+s L_{2}} \\
& v_{m}^{X}(s)=s L_{m}\left(i_{1}(s)+i_{2}(s)\right)-L_{m}\left(i_{1}\left(t_{1}\right)+i_{2}\left(t_{1}\right)\right) .
\end{aligned}
$$

Solving the system given by the equations (10), the three currents in the interval $X$ become:

$$
\begin{aligned}
i_{1}^{X}(t) & =A \cos (\omega t)+B \sin (\omega t) \\
& +\frac{L_{2}}{L_{1}+L_{2}}\left(C \cos \left(\omega_{X} t\right)+D \sin \left(\omega_{X} t\right)\right) \\
i_{2}^{X}(t) & =-A \cos (\omega t)-B \sin (\omega t) \\
& +\frac{L_{1}}{L_{1}+L_{2}}\left(C \cos \left(\omega_{X} t\right)+D \sin \left(\omega_{X} t\right)\right) \\
i_{m}^{X}(t) & =C \cos \left(\omega_{X} t\right)+D \sin \left(\omega_{X} t\right)
\end{aligned}
$$

with

$$
\begin{aligned}
A & =\frac{L_{1}}{L_{1}+L_{2}} i_{1}\left(t_{1}\right)-\frac{L_{2}}{L_{1}+L_{2}} i_{2}\left(t_{1}\right) \\
B & =\frac{V_{1}-V_{2}-v_{C_{1}}\left(t_{1}\right)+v_{C_{2}}\left(t_{1}\right)}{\omega\left(L_{1}+L_{2}\right)} \\
C & =i_{1}\left(t_{1}\right)+i_{2}\left(t_{1}\right) \\
D & =\frac{L_{2}\left(V_{1}-v_{C_{1}}\left(t_{1}\right)\right)+L_{1}\left(V_{2}-v_{C_{2}}\left(t_{1}\right)\right)}{\omega_{X}\left(L_{1} L_{2}+L_{1} L_{m}+L_{2} L_{m}\right)} \\
\omega_{X} & =\sqrt{\frac{1}{\left(\frac{L_{1} L_{2}}{L_{1}+L_{2}}+L_{m}\right)\left(C_{1}+C_{2}\right)}} .
\end{aligned}
$$

By inspection of (11) and (12) the two input subsystems are coupled during the $\mathrm{X}$ interval and the order of the system increases. Eventually, due to this coupling, the use of this nomenclature to analytically derive the power sharing rules has been found forbiddingly complex. Alternatively, a change of variable taking into account the topology is proposed, as detailed in the next section.

\section{Proposed Decoupled Model}

A systematic analytic development, based on changes of variable, that permits to derive power sharing rules is provided in this section. The idea is to obtain a set of variables that are decoupled during all the intervals. Looking at the topology parallel/series symmetries in Fig. 2, the following substitutions in (6) and (11) are proposed

$$
\begin{aligned}
v_{S}(t) & =v_{1}(t)-v_{2}(t) \\
v_{P}(t) & =\frac{L_{2} v_{1}(t)+L_{1} v_{2}(t)}{L_{1}+L_{2}} \\
v_{C S}(t) & =v_{C 1}(t)-v_{C 2}(t) \\
v_{C P}(t) & =\frac{L_{2} v_{C 1}(t)+L_{1} v_{C 2}(t)}{L_{1}+L_{2}} \\
i_{S}(t) & =\frac{L_{1}}{L_{1}+L_{2}} i_{1}(t)-\frac{L_{2}}{L_{1}+L_{2}} i_{2}(t) \\
i_{P}(t) & =i_{1}(t)+i_{2}(t) \\
L_{S} & =L_{1}+L_{2} \\
C_{S} & =\frac{C_{1} C_{2}}{C_{1}+C_{2}} \\
L_{P} & =\frac{L_{1} L_{2}}{L_{1}+L_{2}} \\
C_{P} & =C_{1}+C_{2} .
\end{aligned}
$$




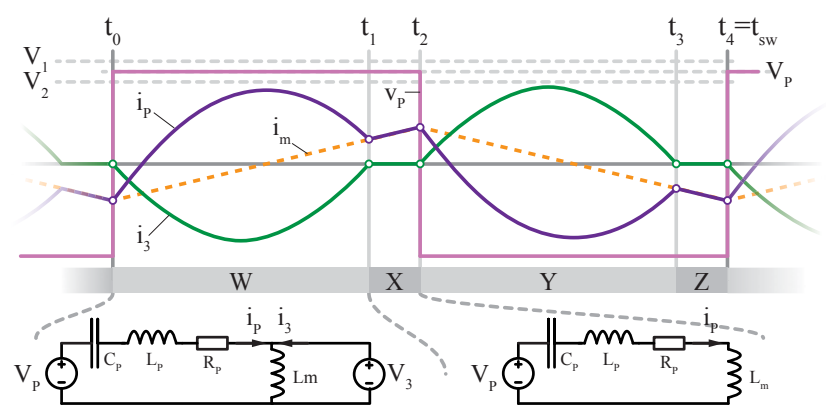

Fig. 4. Common mode equivalent and intervals sub-circuits. Just like in Fig. 3 , each interval corresponds to a simplified equivalent circuit. During interval $W$, the circuit corresponds to (15). The rectifiers are conducting and the voltage on the magnetizing inductor is clamped by $V_{3}$. During interval $X$, the circuit corresponds to (17) and the current $i_{P}$ is equal to the magnetizing current.

After the substitutions, for the interval $W$ :

$$
\begin{aligned}
& i_{S}^{W}(t)=i_{S}\left(t_{0}\right) \cos (\omega t)+\left(\frac{V_{S}-v_{C_{S}}\left(t_{0}\right)}{\omega L_{S}}\right) \sin (\omega t) \\
& i_{P}^{W}(t)=i_{P}\left(t_{0}\right) \cos (\omega t)+\left(\frac{V_{P}-v_{C_{P}}\left(t_{0}\right)-V_{3}}{\omega L_{P}}\right) \sin (\omega t)
\end{aligned}
$$

and for the interval $X$ :

$$
\begin{aligned}
& i_{S}^{X}(t)=i_{S}\left(t_{1}\right) \cos (\omega t)+\left(\frac{V_{S}-v_{C_{S}}\left(t_{1}\right)}{\omega L_{S}}\right) \sin (\omega t) \\
& i_{P}^{X}(t)=i_{P}\left(t_{1}\right) \cos \left(\omega_{X} t\right)+\left(\frac{V_{P}-v_{C_{P}}\left(t_{1}\right)}{\omega_{X}\left(L_{P}+L_{m}\right)}\right) \sin \left(\omega_{X} t\right) .
\end{aligned}
$$

It is worth noting that $i_{S}^{W}(t)$ and $i_{S}^{X}(t)$ can be merged in

$$
i_{S}(t)=i_{S}\left(t_{0}\right) \cos (\omega t)+\left(\frac{V_{S}-v_{C_{S}}\left(t_{0}\right)}{\omega L_{S}}\right) \sin (\omega t)
$$

which holds valid for all intervals defined by $\left(0, t_{s w} / 2\right)$. After the transformation, two fully decoupled $\mathrm{P}$ and $\mathrm{S}$ second order systems are obtained. The sub-circuits that correspond to each circuit and intervals are depicted in Fig. 3 and 4. By inspection of Figs. 3-4, the power delivery to the load depends on the $\mathrm{P}$ circuit, meanwhile the $\mathrm{S}$ circuit only represents power recirculation between the sources. It should be noted that the equivalent circuit $\mathrm{S}$ may also model the variation due to the differential voltage caused by potential differences between the two active ports in terms of switching instants (delays, dead-time, jitter, etc...) whose consequences would affect only circulating current.

\section{POWER Sharing EXPRESSIONS}

As early mentioned, the ideal (lossless) case has been considered firstly, in order to ease the mathematical development. Later, the losses are also included in subsection IV-B, since they have a relevant role in the power sharing characteristics.

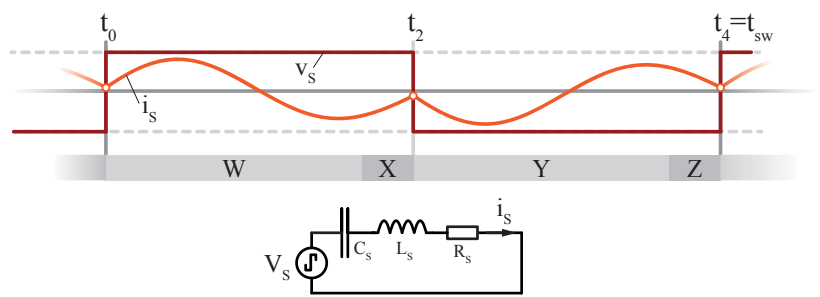

Fig. 5. Differential mode equivalent and intervals sub-circuits. As revealed in (18), the interval expression, as well as the corresponding equivalent circuit, are valid for all both interval $W$ and $X$. In this circuit, only the differential voltage $V_{S}$ and the resonant component (with their series combination $C_{S}$ and $L_{S}$ ) are taken in account and $L_{m}$ does not appear.

\section{A. Ideal Case}

The power delivery to the load is modelled in the circuit $\mathrm{P}$ (cf. Fig. 4), which is defined by

$$
<P_{P}>=\frac{1}{t_{s w}} \int_{0}^{t_{s w}} v_{P}(t) i_{P}(t) d t=\frac{2 V_{P}}{t_{s w}} \int_{0}^{t_{s w} / 2} i_{P}(t) d t .
$$

It should be noticed that circuit $S$ is reactive (cf. Fig. 5), so

$$
\int_{0}^{t_{s w} / 2} i_{S}(t) d t=0
$$

The average power transferred to the load by each active port $(k=1,2)$ is given by

$$
<P_{k}>=\frac{2}{t_{s w}} \int_{0}^{t_{s w} / 2} v_{k}(t) i_{k}(t) d t=\frac{2 V_{k}}{t_{s w}} \int_{0}^{t_{s w} / 2} i_{k}(t) d t .
$$

Development of this expression for the port 1, and also having into account (20) and the changes of variable in (13), gives

$$
\begin{aligned}
<P_{1}> & =\frac{L_{2}}{L_{1}+L_{2}} \frac{2 V_{P}}{t_{s w}} \int_{0}^{t_{s w} / 2} i_{P}(t) d t \\
& +\frac{2 V_{S} L_{1} L_{2}}{t_{s w}\left(L_{1}+L_{2}\right)^{2}} \int_{0}^{t_{s w} / 2} i_{P}(t) d t \\
& =\frac{L_{2}}{L_{1}+L_{2}}<P_{P}>+\frac{L_{1} L_{2}}{\left(L_{1}+L_{2}\right)^{2}} \frac{V_{S}}{V_{P}}\left\langle P_{P}\right\rangle .
\end{aligned}
$$

And, for the second port

$$
<P_{2}>=\frac{L_{1}}{L_{1}+L_{2}}<P_{P}>-\frac{L_{1} L_{2}}{\left(L_{1}+L_{2}\right)^{2}} \frac{V_{S}}{V_{P}}<P_{P}>\text {. }
$$

By inspection of (22) and (23), in a rated condition $V_{S}=V_{1}-V_{2}=0$, the part of the total power that corresponds to each port is a function of the MFT leakage inductances. A deviation from the ratio is caused by the presence of a differential voltage $V_{S}$ (that may be controlled by further control stage), but will, in practice, remain rather small as $V_{S}<<V_{P}$ is expected. At this point, it is worth mentioning that the $\mathrm{P}$ and $\mathrm{S}$ equivalents permit to calculate the power sharing rules without needing to solve the initial conditions of 
the circuit, which significantly eases the analysis. However, the two equivalent circuits $P$ and $S$ being decoupled, the steadystate boundary conditions may be calculated solving:

$$
\begin{aligned}
i_{P}^{W}\left(t_{0}\right) & =-i_{P}^{X}\left(t_{2}\right) \\
v_{C_{P}}^{W}\left(t_{0}\right) & =-v_{C_{P}}^{X}\left(t_{2}\right) \\
i_{S}^{W}\left(t_{0}\right) & =-i_{S}^{X}\left(t_{2}\right) \\
v_{C_{S}}^{W}\left(t_{0}\right) & =-v_{C_{S}}^{X}\left(t_{2}\right)
\end{aligned}
$$

Worst Case Analysis for Tolerances of the Resonant Tank Parameters: If tolerance values are considered for the resonant tank parameters, the assumption of an identical resonant frequency for both resonant tanks, used to get (5), is no longer valid and $\omega_{1}=\sqrt{1 / L_{1} C_{1}} \neq \omega_{2}=\sqrt{1 / L_{2} C_{2}}$. The expressions for power sharing becomes:

$$
\begin{aligned}
<P_{1}> & =\frac{L_{2} \omega_{2}}{L_{1} \omega_{1}+L_{2} \omega_{2}}<P_{P}> \\
& +\frac{L_{1} L_{2} \omega_{1} \omega_{2}}{\left(L_{1} \omega_{1}+L_{2} \omega_{2}\right)^{2}} \frac{V_{S}}{V_{P}}<P_{P}>.
\end{aligned}
$$

And, for the second port

$$
\begin{aligned}
<P_{2}> & =\frac{L_{1} \omega_{1}}{L_{1} \omega_{1}+L_{2} \omega_{2}}<P_{P}> \\
& -\frac{L_{1} L_{2} \omega_{1} \omega_{2}}{\left(L_{1} \omega_{1}+L_{2} \omega_{2}\right)^{2}} \frac{V_{S}}{V_{P}}<P_{P}>.
\end{aligned}
$$

Assuming $V_{s}=0$,

$$
<P_{1}>=\frac{\sqrt{L_{2} / C_{2}}}{\sqrt{L_{2} / C_{2}}+\sqrt{L_{1} / C_{1}}}<P_{P}>.
$$

And, for the second port

$$
<P_{2}>=\frac{\sqrt{L_{1} / C_{1}}}{\sqrt{L_{1} / C_{1}}+\sqrt{L_{2} / C_{2}}}<P_{P}>.
$$

The worst case scenarios correspond to the values that maximize/minimize $\left\langle P_{1}\right\rangle$ and $\left\langle P_{2}\right\rangle$; by inspection of (27)(28) these correspond to the combinations of i) ( $L_{1}^{\min }$, $\left.C_{1}^{\max }, L_{2}^{\max }, C_{2}^{\min }\right)$ and ii) $\left(L_{1}^{\max }, C_{1}^{\min }, L_{2}^{\min }, C_{2}^{\max }\right)$.

\section{B. Consideration of System Losses}

The circuit losses during operation, such as conduction and switching ones, are reflected on the system dynamics and, therefore, can be modeled by equivalent resistors [21]. An a priory estimation of their values is a complex task, but they can be measured from the experimental curves [21]. Equivalent series resistors per port (i.e., $R_{1}$ and $R_{2}$ ) are included in the analysis, with $R_{1} / L_{1} \approx R_{2} / L_{2}$ being assumed. Subsequently, the $\mathrm{P}$ and $\mathrm{S}$ equivalents are defined as

$$
R_{P}=\frac{R_{1} R_{2}}{R_{1}+R_{2}} \quad \text { and } \quad R_{S}=R_{1}+R_{2} .
$$

Figs. 3 and 4 already include these terms. Equation (21) is re-calculated having into account the voltage drops at $R_{P}$ and $R_{S}$ and also having into account that (20) is no longer true (S circuit is not purely reactive) and gives (30). The losses are included within the new terms in (30):

$$
\begin{aligned}
<P_{P_{\text {loss }}}> & =\frac{R_{P}}{t_{s w}} \int_{0}^{t_{s w}} i_{P}^{2}(t) d t \\
<P_{S_{\text {loss }}}> & =\frac{R_{S}}{t_{s w}} \int_{0}^{t_{s w}} i_{S}^{2}(t) d t \\
<P_{S}> & =V_{P} \frac{2}{t_{s w}} \int_{0}^{t_{s w} / 2} i_{S}(t) d t .
\end{aligned}
$$

It has to be noted that the active component of the differential current $i_{S}(t)$ is due to the presence of $R_{S}$.

\section{EXPERIMENTAL RESULTS}

To demonstrate the validity of the developed model and power sharing in practice, a $4 \mathrm{~kW}$ rated converter comprising a three-winding transformer with a 1:1:1 turn ratio is realized. Fig. 6 shows a photography of the lab-scale prototype. The circuit is the same as Fig. 2 with the voltages $V_{1}$ and $V_{2}$ generated by two DC power supplies, allowing to vary precisely the voltage of the two DC buses and test various operating points around $360 \mathrm{~V}$. The MFT is designed to be operated at $12 \mathrm{kHz}$. The load port has a leakage inductance around $0.8 \mu \mathrm{H}$, and the two active ports are equipped with resonant tanks with the parameters presented in Table I. Assuming a 5\% tolerance for the resonant tank components in Table I, the maximum deviations for $P_{1}$ and $P_{2}$ are $6.8 \%$ and $3.4 \%$, respectively. The three half-bridge converters are made of PEB-4046 modules from Imperix [22] which integrates the two IGBTs and the DC-bus capacitors, with the parameters given in Table I. Two of them (on ports 1 and 2) are actively switched at $f_{s w}$ while the third one is passive and used as a rectifier.

The experiment is carried with resistive loads rated to 1,2 , 3 and $4 \mathrm{~kW}$, and for different values of $V_{S}$, between $-15 \mathrm{~V}$ and $25 \mathrm{~V}$. Fig. 7 shows the measured currents waveforms for two different operating points. The powers $P_{1}, P_{2}$ and $P_{3}$ are directly measured at the DC terminals (using a N4L PPA5500 power analyzer). Power analyzer measurements well match calculations computed from the time-domain waveforms (acquired by the oscilloscope, if needed $P_{P}$ and $P_{S}$ are computed from oscilloscope measurements). Fig. 8 depicts the influence of the tank impedance ratio on the sharing of $P_{P}$ between $P_{1}$ and $P_{2}$. The natural sharing characteristic is defined by the tank design and is maintained with changing load on the output (third) port. The losses effects on this result are clear:

TABLE I

EXPERIMENTAL SETUP PARAMETERS

\begin{tabular}{|l|l||l|l|}
\hline Parameter & Value & Parameter & Value \\
\hline$L_{m}$ & $400 \mu \mathrm{H}$ & IGBT & IXYs MMIX1X200N60B3H1 \\
\hline$L_{1}$ & $17.5 \mu \mathrm{H}$ & Deadtime & $800 \mathrm{~ns}$ \\
\hline$C_{1}$ & $5 \mu \mathrm{F}$ & DC-Bus & $825 \mu \mathrm{F}$ \\
\hline$L_{2}$ & $35 \mu \mathrm{H}$ & $f_{\text {res }}$ & $17 \mathrm{kHz}$ \\
\hline$C_{2}$ & $2.5 \mu \mathrm{F}$ & $f_{\text {sw }}$ & $12 \mathrm{kHz}$ \\
\hline
\end{tabular}




$$
\begin{aligned}
& <P_{1}>=\frac{L_{2}}{L_{1}+L_{2}}<P_{P}>+\frac{L_{1} L_{2}}{\left(L_{1}+L_{2}\right)^{2}} \frac{V_{S}}{V_{P}}<P_{P}>+<P_{S}>+\frac{L_{2}}{L_{1}+L_{2}}<P_{P_{\text {loss }}}>+\frac{L_{1}}{L_{1}+L_{2}}<P_{S_{\text {loss }}}> \\
& <P_{2}>=\frac{L_{1}}{L_{1}+L_{2}}<P_{P}>-\frac{L_{1} L_{2}}{\left(L_{1}+L_{2}\right)^{2}} \frac{V_{S}}{V_{P}}<P_{P}>-<P_{S}>+\frac{L_{1}}{L_{1}+L_{2}}<P_{P_{\text {loss }}}>+\frac{L_{2}}{L_{1}+L_{2}}<P_{S_{\text {loss }}}>
\end{aligned}
$$

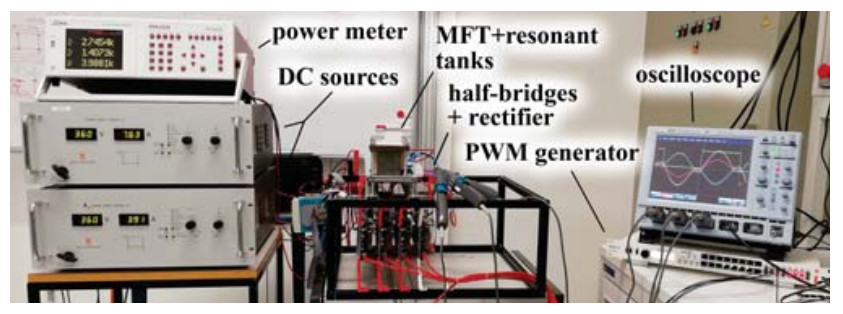

Fig. 6. $4 \mathrm{~kW} / 360 \mathrm{~V}$ rated experimental test-setup.

when the output power increases, the weight of losses terms in (30) is smaller and, therefore, (22)-(23) are more accurate. However, when $P_{P}$ is small, all the terms of (30) have an impact. In order to deep into the accuracy of the model with losses consideration, Figs. 9 and 10 are assessed. The effect of $V_{S}$ on $P_{S}$ is shown in Fig. 9, illustrating that for the constant output load, the power sharing is highly sensitive to variation of $V_{S}$. The impact of $V_{S}$ on the efficiency is depicted in Fig. 10 for the different loads although the lab set-up hardware has not been optimized in that sense, and its purpose is to validate power sharing characteristics.

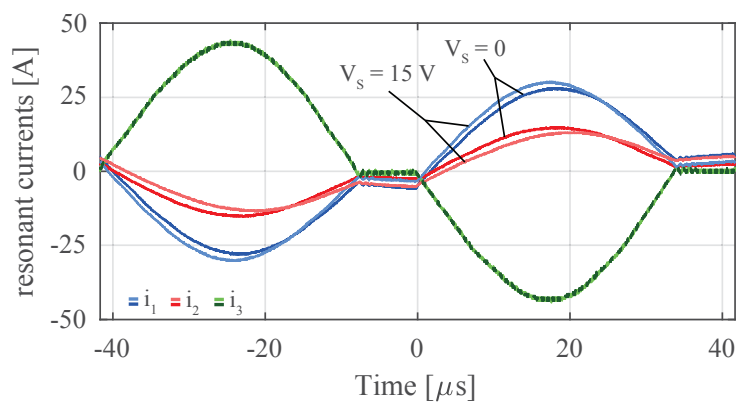

Fig. 7. Resonant current waveforms (oscilloscope import) from measurement at $4 \mathrm{~kW}$. The currents $i_{1}$ and $i_{2}$ are affected by the introduction of differential voltage $V_{S}$, but the current to the load, namely $i_{3}$ is not affected. To be noted that from $t=-40 \mu \mathrm{s}$ to 0 , the currents flow in $S_{1 L}, S_{2 L}$ and $D_{3 L}$ while from $t=0$ to $40 \mu \mathrm{s}$, the currents circulate in $S_{1 H}$ and $S_{2 H}$ and $D_{3 H}$

\section{CONCLUSION}

This paper presents the model and the power sharing characteristics, for a DC-transformer operated three-port DCDC converter when two actively switching ports deliver power to a load connected at the third one. In order to get a compact analytic solution, a model based the separation of the power flow into a main power (from the active ports to the load port) and a circulating power (between the active ports) is proposed. It reveals that the power sharing is highly impacted by the
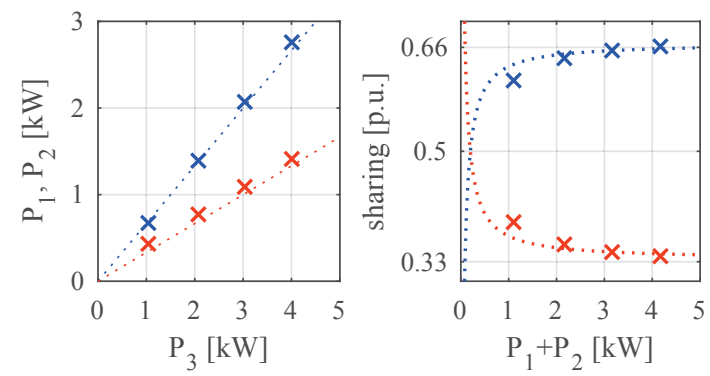

Fig. 8. On the left hand side, the powers ( $P_{1}$ in blue and $P_{2}$ in red $)$ and the theoretical value (dashed) in the lossless case. On the right hand side, their corresponding part of $\left(P_{1}+P_{2}\right)$ for $V_{S}=0$. Apart from some deviations for light load conditions, the power sharing is constant and reflecting the ratio of the inductance defined in Table I, namely $L_{2} /\left(L_{1}+L_{2}\right)=2 / 3$ and $L_{1} /\left(L_{1}+L_{2}\right)=1 / 3$. At low power operation, the impact of the switching losses which are load independent (offset) and are not precisely modelled here is more important compared to the conduction losses effect and their sharing may diverge from the ratio specified above. The dashed lines show the theoretical sharing in the case of presence of $40 \mathrm{~W}$ losses in no load conditions.

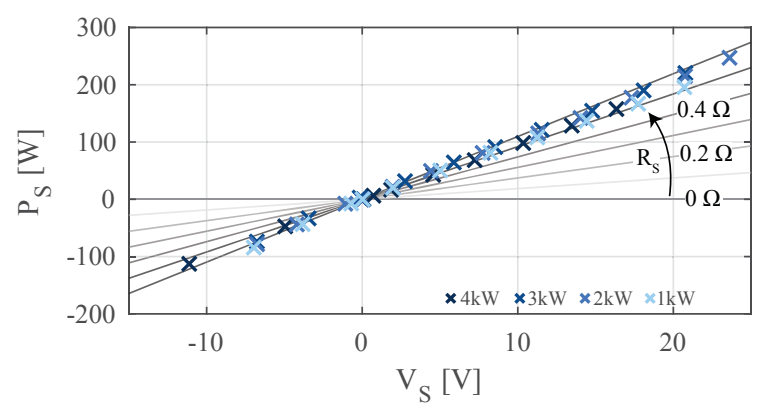

Fig. 9. Measured $P_{S}$ and effect of $R_{S}$ on the theoretical curve. For $R_{S}=0$, it would be zero for any $V_{S}$. The experiments show that $R_{S}$, which represent the conduction losses and the switching losses associated to the circulating power flow, is around $0.55 \Omega$.

resonant tank design parameters which allows any desired ratio to be achieved by choosing the appropriate resonant tank inductances. The sharing is preserved in absence of differential voltage between the active ports and can be, to some extent, influenced by further manipulation on the differential voltage which introduces a circulating power flow (influenced as well by the losses and parasitic resistances). The power sharing dependence on the input voltages would establish the plant modelling if power sharing controllers are considered in the additional power stages of a MEG structure (e.g., blue blocks of Fig. 1). The experimental results, obtained with a lab-scale prototype, match and effectively verify the proposed model. 


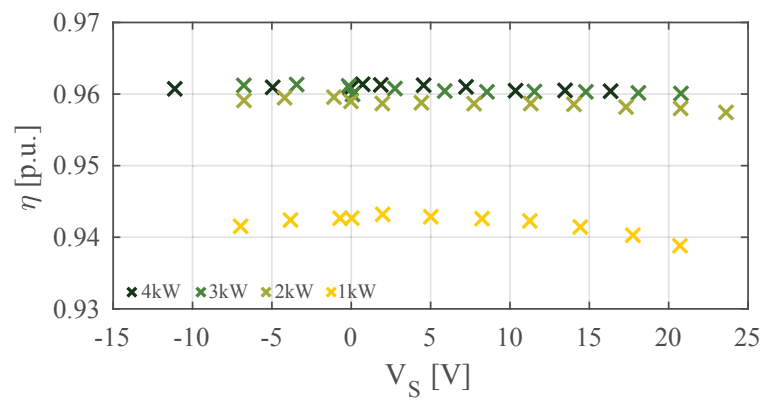

Fig. 10. Efficiency for various operating points. The switching losse depending on the turn off currents $\left(i_{1}(0)\right.$ and $\left.i_{2}(0)\right)$ which are set by $L_{m}$ and are load independent, the efficiency is reduced for light load conditions. Additional losses appear with the circulation of current $i_{S}$, which explain the drop of efficiency for increasing $\left|V_{S}\right|$

\section{ACKNOWLEDGEMENT}

This research project is part of the National Research Program "Energy Turnaround" (NRP70) of the Swiss National Science Foundation (SNSF). Further information on the National Research Program can be found at www.nrp70.ch.

\section{REFERENCES}

[1] G. Bathurst, G. Hwang, and L. Tejwani, "Mvdc - the new technology for distribution networks," in 11th IET International Conference on AC and DC Power Transmission, Feb 2015, pp. 1-5.

2] U. Javaid, D. Dujic, and W. van der Merwe, "Mvdc marine electrical distribution: Are we ready?" in Proc. IEEE IECON, Nov 2015, pp. 000 823-000 828.

[3] Z. Jin, G. Sulligoi, R. Cuzner, L. Meng, J. C. Vasquez, and J. M. Guerrero, "Next-generation shipboard dc power system: Introduction smart grid and dc microgrid technologies into maritime electrical netowrks,' IEEE Electrification Magazine, vol. 4, no. 2, pp. 45-57, June 2016.

[4] H. Choi, M. Ciobotaru, M. Jang, and V. G. Agelidis, "Performance of medium-voltage dc-bus pv system architecture utilizing high-gain dc-dc converter," IEEE Transactions on Sustainable Energy, vol. 6, no. 2, pp 464-473, April 2015

[5] C. Zhan, C. Smith, A. Crane, A. Bullock, and D. Grieve, "Dc transmission and distribution system for a large offshore wind farm," in $A C$ and DC Power Transmission, 2010. ACDC. 9th IET International Conference on, Oct 2010, pp. 1-5.

[12] Y. K. Tran and D. Dujic, "A multiport medium voltage isolated dc-dc converter," in Proc. IEEE IECON, Oct 2016, pp. 6983-6988.
[6] "Dc for efficiency," $A B B$ Review 4/13, 2013. [Online]. Available: https://library.e.abb.com/public/60289ad65efef903c1257c5a0040a37b/ 16-21\\%204m412_EN_72dpi.pdf

[7] M. Stieneker and R. W. D. Doncker, "Medium-voltage dc distribution grids in urban areas," in 2016 IEEE 7th International Symposium on Power Electronics for Distributed Generation Systems (PEDG), June 2016, pp. 1-7.

[8] Y. Tang and A. Khaligh, "Bidirectional hybrid battery/ultracapacitor energy storage systems for next generation mvdc shipboard power systems," in Vehicle Power and Propulsion Conference (VPPC), 2011 IEEE, Sept 2011, pp. 1-6.

[9] H. Tao, A. Kotsopoulos, J. Duarte, and M. Hendrix, "Family of multiport bidirectional dc-dc converters," Electric Power Applications, IEE Proceedings -, vol. 153, no. 3, pp. 451-458, May 2006.

[10] D. Dujic, C. Zhao, A. Mester, J. K. Steinke, M. Weiss, S. LewdeniSchmid, T. Chaudhuri, and P. Stefanutti, "Power electronic traction transformer-low voltage prototype," IEEE Trans. Power Electron. vol. 28, no. 12, pp. 5522-5534, 2013.

[11] C. Zhao et al., "Power electronic traction transformer - medium voltage prototype," IEEE Trans. Ind. Electron., vol. 61, no. 7, pp. 3257-3268, July 2014.

[13] C. Gu, Z. Zheng, and Y. Li, "A power electronic transformer (pet) with multiport bidirectional resonant dc-dc converters for electric traction applications," in 2015 IEEE Transportation Electrification Conference and Expo (ITEC), June 2015, pp. 1-6.

[14] Z. Pavlovic, J. A. Oliver, P. Alou, O. Garcia, and J. A. Cobos, "Bidirectional multiple port dc/dc transformer based on a series resonan converter," in Proc. IEEE APEC, March 2013, pp. 1075-1082.

[15] K. Tomas-Manez, Z. Zhang, and Z. Ouyang, "Multi-port isolated llc resonant converter for distributed energy generation with energy storage,' in Proc. IEEE ECCE, Oct 2017, pp. 2219-2226.

[16] Y. K. Tran and D. Dujic, "A multiport isolated dc-dc converter," in Proc. IEEE APEC, March 2016, pp. 156-162.

[17] Y. K. Tran, D. Dujic, and P. Barrade, "Multiport resonant dc-dc converter," in Proc. IEEE IECON, Nov 2015, pp. 3839-3844.

[18] C. Oeder and T. Duerbaum, "Zvs investigation of LLC converters based on fha assumptions," in Proc. IEEE APEC, March 2013, pp. 2643-2648.

[19] M. A. Halim, M. N. Seroji, and M. N. B. Hidayat, "Analysis characteristics and optimal design procedure of half-bridge llc loaded resonant converter," in 2012 IEEE International Conference on Power and Energy (PECon), Dec 2012, pp. 564-569.

[20] D. Rothmund, J. E. Huber, and J. W. Kolar, "Operating behavior and design of the half-cycle discontinuous-conduction-mode series-resonantconverter with small dc link capacitors," in 2013 IEEE 14th Workshop on Control and Modeling for Power Electronics (COMPEL), June 2013, pp. $1-9$.

[21] A. Vidal et al., "A method for identification of the equivalent inductance and resistance in the plant model of current-controlled grid-tied converters," IEEE Trans. Power Electron., vol. 30, no. 12, pp. 7245-7261, Dec 2015.

[22] "Imperix ltd," https://imperix.ch/products/power/peb 